\title{
A COMPARISON OF PATIENTS WITH HIP FRACTURE, TEN YEARS APART: MORBIDITY, MALNUTRITION AND SARCOPENIA
}

\author{
N. PROBERT ${ }^{1,2}$, A. LÖÖW ${ }^{3}$, G. AKNER ${ }^{4}$, P. WRETENBERG ${ }^{1,5}$, Å.G. ANDERSSON 1,6
}

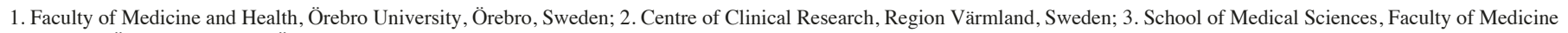
and Health, Örebro University, Örebro, Sweden; 4. Department of Neurobiology, Care Sciences and Society, Karolinska Institutet, Stockholm, Sweden; 5. Department of Orthopaedics,

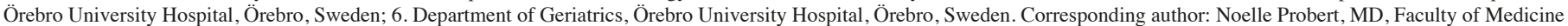
and Health, Örebro University, Örebro, Sweden, noelle.probert@oru.se

\begin{abstract}
Objectives: To investigate possible differences in morbidity, malnutrition, sarcopenia and specific drug use in patients with hip fracture, ten years apart. To analyse 1-year mortality and possible associations with variables. Design: A prospective, observational study. Setting: Örebro University Hospital, Sweden. Participants: Two cohorts of patients with hip fracture, included in $2008(\mathrm{n}=78)$ and $2018(\mathrm{n}=76)$. Measurements: Presence of comorbidity according to the Elixhauser comorbidity measure, multimorbidity defined as $\geq 3$ comorbidities, preoperative American Society of Anaesthesiologists Classification (ASA-class), malnutrition according to the definition by the Global Leadership Initiative on Malnutrition (GLIM), sarcopenia according to the most recently revised definition by the European Working Group on Sarcopenia in Older People (EWGSOP), polypharmacy defined as $\geq 5$ prescribed medications, use of Potentially Inappropriate Medications (PIM) and Fall-Risk-Increasing-Drugs (FRID) and postoperative 1-year mortality. Results: When comparing the cohorts, significant increases over time was seen for mean comorbidity-count (Difference -1; $\mathrm{p}=0.002$ ), multimorbidity (Difference -15\%; 95\%CI -27;-2), ASA-class 3-4 (Difference -25\%; 95\%CI -39;-9) and polypharmacy (Difference $-17 \%$; 95\%CI -32;-2). Prevalence of malnutrition and sarcopenia coherently decreased with $22 \%$ (95\% CI 5;37) and 14\% (95\% CI 1;29) respectively. One-year mortality remained unchanged and a significant association was found for a higher ASA-class in 2008 (OR 3.5, 95\%CI 1.1;11.6) when adjusted for age. Results on PIM exposure suggest a decrease while exposure to FRID remained high. Conclusion: Our findings support an increasing morbidity within the population over time. However, also presented is a coherent decrease in malnutrition and sarcopenia, suggesting a decrease in frailty as a possible explanation for the observed unaltered mortality, in turn suggesting advances in treatment of comorbidities.
\end{abstract}

Key words: Hip fracture, comorbidity, malnutrition, sarcopenia, mortality.

\section{Introduction}

Hip fracture primarily affects older people and low-energy trauma is the most common cause due to osteoporosis and an increased risk of falling. According to Swedish national data the mean age at time of fracture is 82 years and $67 \%$ of the patients are of female gender (1). Sweden represents one of the highest incidences worldwide with close to 17.000 cases annually $(1,2)$. The incidence of hip fracture is estimated to escalate as people live longer (3), a major concern due to the following economic burden, poor outcome and excess mortality (4-6), 1-year mortality-rate amounting to $>25 \%$ in Sweden (7).

Patients typically suffer from a high premorbid frailty, multimorbidity and polypharmacy, factors found to increase risk of hip fracture (8-10). Malnutrition, sarcopenia and comorbidity, overlapping with- and contributing to frailty, being a multifactorial clinical condition $(11,12)$, are factors associated with an increased mortality post fracture (13-15). Consensus has recently been reached regarding a definition of malnutrition by the Global Leadership Initiative on Malnutrition (GLIM) (16). Recommendations on defining sarcopenia have also latterly been revised by the European Working Group on Sarcopenia in Older People (EWGSOP) (17). Two major categories of drugs are frequently mentioned in studies. Older people and particularly patients with hip fracture have a vulnerability to Potentially Inappropriate Medications (PIM), associated with increased mortality postfracture (18). Fall-Risk-Increasing-Drugs (FRID), prevalent within the population, increase hip fracture risk and are also associated with an increased mortality $(19,20)$. Due to their observed adverse events in older people, several international lists of PIM and FRID have been established in order to increase awareness.

Contradictory to earlier estimates, hip fracture incidence is declining and Swedish data suggests that coherent survival rates have remained unaltered (21), possibly explained by a potential change in morbidity of the population. A few previous studies have examined the development of the population and its morbidity over time and present homogenous results of an increased comorbidity-burden and polypharmacy while mortality has decreased or remained unchanged, possibly portraying advances in treatment of comorbidities, hip fracture and individualized care (22-27). In light of this there is a value in studying how a possible increase in morbidity may reflect possible changes in malnutrition and sarcopenia as well as specific drug use, to our knowledge not yet studied. 


\section{THE JOURNAL OF NUTRITION, HEALTH \& AGING}

\section{Aim}

The primary aim was to investigate possible differences in morbidity, malnutrition, sarcopenia and specific drug use in patients with hip fracture, ten years apart. Our secondary aim was to analyse 1-year mortality and possible associations with variables.

\section{Methods}

\section{Study design and population}

In this prospective, observational cohort study all patients undergoing surgery at Örebro University Hospital due to hip fracture diagnosed with ICD-10 codes S72.0, S72.1 or S72.2 during 5 months in 2008 and in 2018 respectively, were consecutively invited to participate. No exclusion criteria existed.

\section{Morbidity and drugs}

Data on diseases, ASA-class (28) and medications was obtained from individual medical records. Diseases were verified according to ICD-10, all Elixhauser comorbidities were evaluated $(29,30)$. Multimorbidity was defined as $\geq 3$ comorbidities. Polypharmacy and excessive polypharmacy was defined as $5-9$ and $\geq 10$ prescribed medications respectively.

PIM were identified from indicator 1.1 (drugs that should be avoided if explicit reasons for prescription do not apply) of the drug specific indicators compiled by the Swedish National Board of Health and Welfare (SNBHW) (31) and a list (drugs that should be prescribed restrictively) compiled by the Drug and Therapeutics Committee of Örebro County (32). Drugs defined as FRID were identified from indicator 1.8 (drugs and specific symptoms; drugs that increase the risk of falling) by the SNBHW (31) and a list (drugs that can increase the risk of falling) compiled by the Drug and Therapeutics Committee of Örebro County (32). Included drugs can be viewed in Supplementary Dataset S1.

\section{Malnutrition and sarcopenia}

Anthropometric measurements were obtained through clinical bedside examinations.

Malnutrition was diagnosed according to GLIM-criteria (16): At least one phenotypic (listed below) and one etiologic (decreased food intake or inflammatory condition/disease burden) criterion has to be met for diagnosis. Hip fracture was considered an etiologic criterion (16). Phenotypic criteria consist of:

- Low BMI $(\mathrm{kg} / \mathrm{m} 2)$, cut-off $<20$ if $<70$ years or $<22$ if $>70$ years (16).

- Reduced muscle mass, measured as calf circumference (CC), cut-off $<31 \mathrm{~cm}$ (33).

- Non-volitional weight loss the last three months, measured by the screening-tool Mini Nutritional Assessment (34).
Documentation on weight loss was very poor and therefore excluded from possible phenotypic criteria. Patients were thus considered malnourished if they had low BMI or CC under cutoff in addition to hip fracture as the etiologic criteria.

Sarcopenia was diagnosed according to EWGSOP2-criteria (17), consisting of the following three steps:

- Reduced muscle strength indicating probable sarcopenia. Measured as hand-grip strength using a hand dynamometer, the best attempt of three on the best hand was evaluated, cutoff $<27 \mathrm{~kg}$ for men and $<16 \mathrm{~kg}$ for women (35).

- Reduced muscle mass confirming diagnosis, measured as CC, cut-off $<31 \mathrm{~cm}(17)$.

- Impaired physical performance determining severity; not evaluated in this study.

\section{Statistics}

Differences in mean age, length of stay, comorbidity, BMI, $\mathrm{CC}$ and hand grip strength was analysed by independent sample t-test. Differences in gender was analysed by chi-squared test. Level of statistical significance was set at $p<0.05$. Differences in proportions for dichotomized variables were calculated with the method described by Newcombe \& Altman (36). Differences in proportions are presented as $95 \%$ confidence intervals, the interval will be significant if it does not include zero.

Odds ratios adjusted for age were calculated by logistic regression analysis, the $95 \%$ confidence interval will be significant if it does not include one.

The t-test, chi-squared test and calculation of odds ratios were performed in SPSS Statistics 25. Differences in proportions were calculated with the software program Confidence Interval Analysis.

\section{Results}

\section{Participants}

In total, 108 patients in 2008 and 97 in 2018 were invited to participate where 30 and 21 patients were unable to, respectively, leaving 78 patients included in 2008 and 76 in 2018. The major reason for non-inclusion was impaired ability to give consent due to cognitive state.

When comparing dropout groups with participants there was no significant difference in gender, in $2008(\mathrm{p}=0.96)$ or 2018 $(\mathrm{p}=0.70)$. In 2008 there was no significant difference in mean age $(\mathrm{p}=0.26)$, the drop-out group presenting a mean age of 84 years compared to 81 among participants, whereas in 2018, the dropout group presented a significantly higher mean age of 87 compared to 80 among participants ( $\mathrm{p}=0.007$ ).

\section{Baseline characteristics}

Patients were similar regarding baseline characteristics (table 1), there were no significant differences in mean age or gender distribution. Pre-fractural housing and prevalence of walking aids was similar. 
Table 1

Baseline characteristics of the two cohorts of patients with hip fracture

\begin{tabular}{|c|c|c|c|}
\hline & Cohort $2008 \mathrm{n}=78$ & Cohort $2018 n=76$ & Difference, [p-value] / (95\% CI) \\
\hline Age, mean (SD) & $81(11)$ & $80(12)$ & -1 year \\
\hline Min-max & $35-98$ & $41-103$ & {$[0.55]$} \\
\hline Female, n (\%) & $49(63)$ & $47(62)$ & $-1(-14 ; 16)$ \\
\hline Ordinary housing, n (\%) & 69 of 77 (90) & $69(91)$ & $-1(-11 ; 9)$ \\
\hline Living alone before fracture, $\mathrm{n}(\%)$ & $45(58)$ & $48(63)$ & $-5(-20 ; 10)$ \\
\hline Walking aid before fracture, $\mathrm{n}(\%)$ & 33 of $76(43)$ & $36(47)$ & $-4(-19 ; 12)$ \\
\hline Length of stay, mean (SD) & $10(5)$ & $9(4)$ & $1[0.58]$ \\
\hline Coplanar fall-related fracture, n (\%) & $76(97)$ & $71(93)$ & $4(-3 ; 12)$ \\
\hline Fall indoors, $\mathrm{n}(\%)$ & 57 of $71(80)$ & 53 of $73(73)$ & $7(-6 ; 21)$ \\
\hline \multicolumn{4}{|l|}{ Type of fracture, n (\%): } \\
\hline $\mathrm{S} 72.0^{\mathrm{a}}$ & $41(53)$ & $37(49)$ & $4(-12 ; 19)$ \\
\hline $\mathrm{S} 72.1^{\mathrm{b}}$ & $31(40)$ & $31(41)$ & $-1(-16 ; 14)$ \\
\hline $\mathrm{S} 72.2^{\mathrm{c}}$ & $6(8)$ & $8(11)$ & $-3(-13 ; 7)$ \\
\hline
\end{tabular}

Abbreviations: CI, confidence interval; SD, standard deviation; a. Femoral neck fracture; b. Subtrochanteric femoral fracture; c. Pertrochanteric femoral fracture.

\section{Figure 1 A-B}

A. Differences in prevalence of malnutrition, sarcopenia, low BMI, weight loss, calf circumference under cut-off and grip strength under cut-off comparing the two cohorts of patients with hip fracture. B. Differences in mean values of body mass index, calf circumference and grip strength comparing the two cohorts of patients with hip fracture

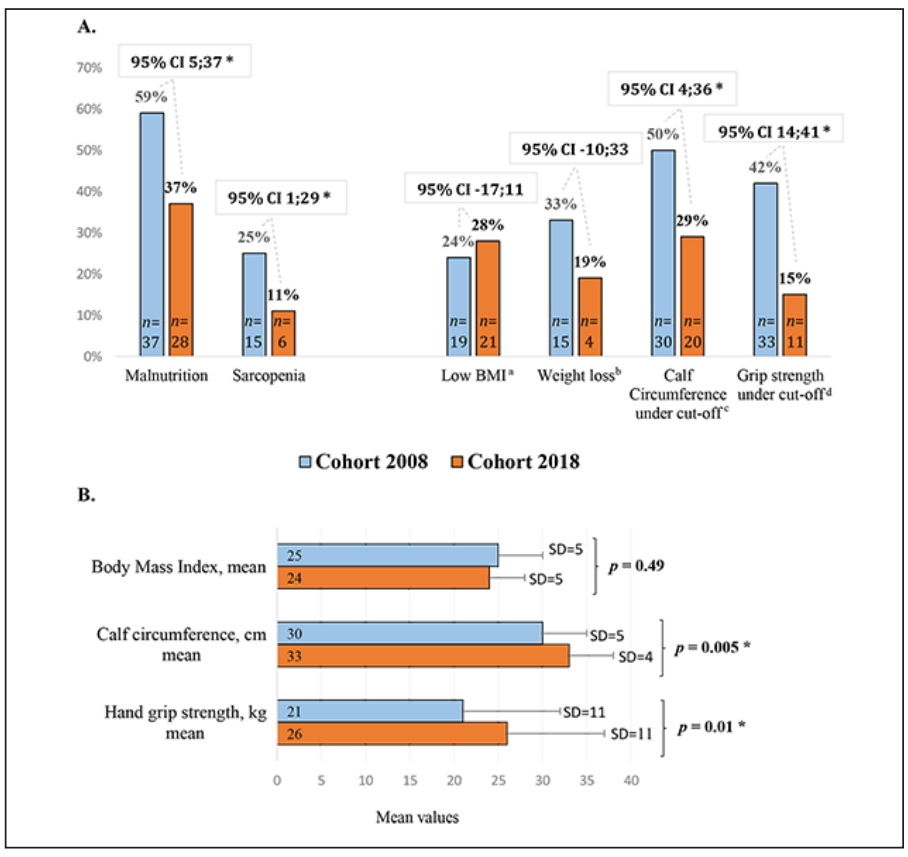

Abbreviations: CI, confidence interval; BMI, body mass index; SD, standard deviation $\mathrm{a}, \mathrm{kg} / \mathrm{m} 2,<20$ if $<70$ years or $<22$ if $>70$ years; b, During the last 3 months; c, $<31 \mathrm{~cm}$; d, measured with a hand dynamometer, $<27 \mathrm{~kg}$ for men and $<16 \mathrm{~kg}$ for women; *, significant.
Figure 2 A-B

A. Possible associations of variables with 1-year mortality post hip fracture surgery in 2008, presented in a forest plot as odds ratios and $95 \%$ confidence intervals adjusted for age. B. Possible associations of variables with 1-year mortality post hip fracture surgery in 2018 , presented in a forest plot as odds ratios and $95 \%$ confidence intervals adjusted for age
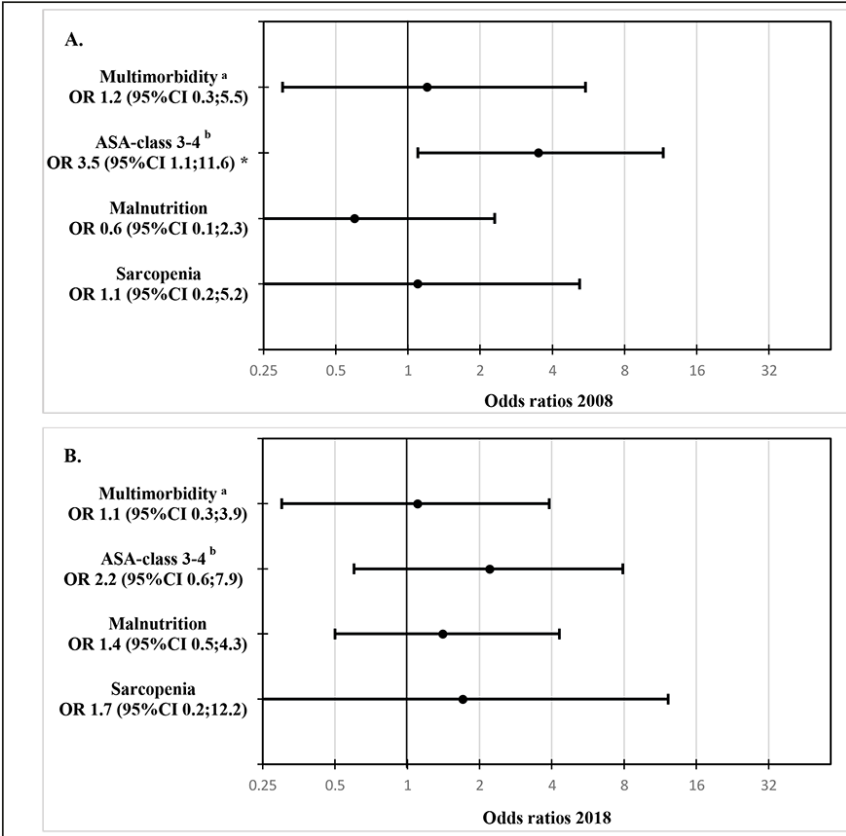

Abbreviations: OR, odds ratio; CI, confidence interval; ASA-class, American Society of Anaesthesiologists classification. a, Multimorbidity defined as having $\geq 3$ comorbidities of the Elixhauser comorbidity measure. b, Ranging from 1-6, no patients were assessed with an ASA-class $>4$; *, significant. 
Table 2

Differences in morbidity, polypharmacy and exposure to PIM and FRID between the two cohorts of patients with hip fracture

\begin{tabular}{|c|c|c|c|}
\hline & $\begin{array}{c}\text { Cohort 2008, } \\
n=78\end{array}$ & $\begin{array}{c}\text { Cohort 2018, } \\
n=76\end{array}$ & $\begin{array}{c}\text { Difference, } \\
\text { [p- value]/ }(95 \% \text { CI })\end{array}$ \\
\hline Multimorbidity ${ }^{\mathrm{a}}, \mathrm{n}(\%)$ & $10(13)$ & $21(28)$ & $-15(-27 ;-2) *$ \\
\hline ASA-class 3 and 4,n (\%) & 27 of 75 (36) & $46(61)$ & $-25(-39 ;-9) *$ \\
\hline Comorbidity, mean (SD) & $1(1)$ & $2(1)$ & $-1[0.002] *$ \\
\hline Congestive heart failure & $10(13)$ & $11(15)$ & $-2(-13 ; 9)$ \\
\hline Cardiac arrythmia & $13(17)$ & $18(24)$ & $-7(-20 ; 6)$ \\
\hline Valvular disease & $3(4)$ & $5(7)$ & $-3(-11 ; 5)$ \\
\hline Peripheral vascular disorders & $0(0)$ & $3(4)$ & $-4(-11 ; 1)$ \\
\hline Hypertension, uncomplicated & $20(26)$ & $42(55)$ & $-29(-43 ;-14) *$ \\
\hline Neurological disorder & $6(8)$ & $4(5)$ & $3(-6 ; 11)$ \\
\hline Paralysis & $1(1)$ & $1(1)$ & $0(-6 ; 6)$ \\
\hline Chronic pulmonary disease & $9(12)$ & $8(11)$ & $1(-9 ; 11)$ \\
\hline Diabetes, uncomplicated & $6(8)$ & 7 (9) & $-1(-11 ; 8)$ \\
\hline Diabetes, complicated & $0(0)$ & $4(5)$ & $-5(-13 ; 0)$ \\
\hline Hypothyroidism & $4(5)$ & $6(8)$ & $-3(-12 ; 6)$ \\
\hline Renal failure & $1(1)$ & 7 (9) & $-8(-17 ;-1) *$ \\
\hline Liver disease & $0(0)$ & $3(4)$ & $-4(-11 ; 1)$ \\
\hline Tumour & $6(8)$ & $6(8)$ & $-0(-9 ; 9)$ \\
\hline Lymphoma & $1(1)$ & $0(0)$ & $1(-4 ; 7)$ \\
\hline Metastatic cancer & $1(1)$ & $1(1)$ & $0(-6 ; 6)$ \\
\hline Rheumatoid arthritis & $3(4)$ & $2(3)$ & $1(-6 ; 8)$ \\
\hline Coagulopathy & $1(1)$ & $0(0)$ & $1(-4 ; 7)$ \\
\hline Alcohol abuse & $0(0)$ & $2(3)$ & $-3(-9 ; 2)$ \\
\hline Depression & $0(0)$ & $2(3)$ & $-3(-9 ; 2)$ \\
\hline Polypharmacy, $\geq 5$ drugs, n (\%) & 40 of $77(52)$ & 52 of $75(69)$ & $-17(-32 ;-2) *$ \\
\hline Excessive polypharmacy, $\geq 10$ drugs, $\mathrm{n}(\%)$ & 11 of 77 (14) & 16 of $75(21)$ & $-7(-19 ; 5)$ \\
\hline Number of patients exposed to at least one PIM, n (\%) & 15 of $77(20)$ & 11 of $75(15)$ & $5(-7 ; 17)$ \\
\hline Number of patients exposed to at least one FRID, n (\%) & 63 of $77(82)$ & 62 of $75(83)$ & $-1(-13 ; 12)$ \\
\hline
\end{tabular}

\section{Morbidity, malnutrition, sarcopenia and drug use}

In total, there were 85 comorbidities in 2008 and 133 in 2018. Cohort 2018 presented significantly higher figures of comorbidity, multimorbidity, and ASA-class 3-4. No patients were assessed with an ASA-class higher than 4. Significant differences were seen for the individual comorbidities of uncomplicated hypertension and renal failure, more prevalent in 2018. Pulmonary circulation disorders, complicated hypertension, peptic ulcer disease, AIDS/HIV, blood loss anaemia, fluid and electrolyte disorders, weight loss, obesity, psychoses and drug abuse were not prevalent at all and thus not included in table 2 .
Polypharmacy was significantly more prevalent in 2018 . Results indicate a decrease in PIM-exposure while exposure to FRID remained high. In both cohorts, the most common PIM-categories were hypnotics and sedatives followed by anticholinergics and the most common FRID-categories were cardiovascular FRID followed by psychotropics. Zolpidem was the most frequently prescribed PIM, 10 patients exposed in 2008 and 5 in 2018. In 20083 patients were prescribed the PIM Tramadol, not prevalent in 2018. The most common FRID were: Furosemide, Metoprolol, and Zolpidem in 2008 and Furosemide, Metoprolol and Amlodipine in 2018.

Prevalence of malnutrition and sarcopenia was significantly 
Table 3

One-year mortality post-surgery for hip fracture of the two cohorts and possible associations with variables. Odds ratios and $95 \%$ confidence intervals adjusted for age

\begin{tabular}{|c|c|c|c|c|c|c|c|c|c|c|}
\hline & \multicolumn{5}{|c|}{ Cohort $2008 n=78$} & \multicolumn{5}{|c|}{ Cohort 2018 n=76 } \\
\hline ASA-class 3-4 b & 10 & 16 & 17 & 59 & $3,5(1.1 ; 11.6) *$ & 13 & 17 & 33 & 59 & 2,2 (0.6;7.9) \\
\hline Malnutrition & 6 & 11 & 31 & 52 & $0,6(0.1 ; 2.3)$ & 8 & 17 & 20 & 58 & $1,4(0.5 ; 4.3)$ \\
\hline
\end{tabular}

Abbreviations: OR, Odds ratio; CI, confidence interval; ASA-class, American Society of Anaesthesiologists Classification; a. Multimorbidity defined as having $\geq 3$ comorbidities of the Elixhauser comorbidity measure; b. Ranging from 1-6, no patients were assessed with an ASA-class $>4$. *, significant.

higher in 2008, coinciding with more patients having a grip strength and $\mathrm{CC}$ under cut-off (figure 1A). In line with this, cohort 2018 presented significantly higher values of mean CC and grip strength than cohort 2008 (figure 1B). BMI and weight-loss did not differ significantly.

\section{Mortality}

One-year mortality remained unaltered with a rate of $23 \%$ in 2008 and $22 \%$ in 2018 (95\% CI -13;14). A logistic regression analysis of associations between variables and 1-year mortality was performed (Table 3 and figure 2A-B.), all odds ratios were adjusted for age. Malnutrition and sarcopenia did not present any significant associations with 1-year mortality. For patients with ASA classification 3-4, there was a significant association with 1-year mortality in 2008 (95\% CI 1.1;11.6) but not in 2018 .

\section{Discussion}

\section{Main findings}

When comparing two cohorts of patients with hip fracture from 2008 and 2018, we found a significant increase in morbidity in terms of comorbidity, preoperative ASA-class and polypharmacy. To the contrary, 1-year mortality remained subsequently unaltered and prevalence of malnutrition and sarcopenia significantly decreased.

\section{Comorbidity and drug use}

In consensus with others, patients were of higher age and to a greater extent of female gender. Cohort 2018 presented a significantly higher morbidity in terms of mean comorbiditycount, multimorbidity and preoperative ASA-class. A few previous studies have also compared the population over time and results of a general increase in comorbidity-burden coincide with ours $(22,24-27)$. Multimorbidity increased from 13 to $28 \%(95 \%$ CI $-27 ;-2)$, comparable with the increase of 33.9 to 43.3\% found in a large observational study between 2000 and 2016 in USA by Bekeris et al, using the same definition of multi- and comorbidity (25). Significant differences regarding individual comorbidities could only be seen for hypertension and renal failure in this study, suggesting an increase. Although, the potential increase of complicated diabetes mellitus was close to significant (Difference -5\%; 95\% CI -13;0). These results are supported by Bekeris et al, however, this author found the largest increases in sleep apnea, not reported on in our study as well as in drug abuse, weight loss and obesity, not prevalent in our results (25). This could be attributable to differences in study-sample and design as well as in healthcare and lifestyle between USA and Sweden. Some comorbidities of the Elixhauser comorbidity measure were not prevalent at all in our results, possibly also attributable to small samplesize. Trevisan et al. preformed similarly to us a smaller study comparing a cohort from 2000 with a cohort from 2015 in Italy and also found significant increases in renal disease in addition to alzheimers, COPD, and valvulopathy (26). Significant increases in renal disease, cardiovascular disease and diabetes have also been reported in the larger longitudinal studies by Jantzen et al. from 1999-2012 in Denmark (24), Baker et al. from 2000-2012 in England (27) and Brauer et al from 19862005 in USA (22).

Evidently there seems to have been a shift towards increased comorbidity over time. However, there is no way of disregarding possible influences of increased screening, awareness and diagnosis. Also supporting our results though is the coherent increase of ASA-class 3-4 of 25\% (95\% CI -39; -9), corresponding to preoperative severe systemic disease (28). These findings resemble the increase of $20 \%$ found in a population-based study by Turesson et al., observing patients between 1999-2017 in Sweden (37). There are however limitations to ASA-class, the scoring scheme for estimation was revised in 2014, with a re-introduction of case-vignettes (28), possibly affecting assessment. Additionally, the subjective assessment of ASA-class is also a limiting factor, we have tried to diminish this by grouping the ASA-scores of 1-2 and 3-4.

A potential reason for increasing comorbidity could be increasing age of the patients (38). This study presented no significant difference regarding this and results of other studies are inconclusive, Trevisan et al. and Brauer et al. found significant increases in age over 90 and 85 respectively while 


\section{THE JOURNAL OF NUTRITION, HEALTH \& AGING}

other studies support our findings $(24,25,27)$. Numerous individual diseases have been associated with increased risk of hip fracture (10), therefore a coherent increase in incidence could be expected due to current results on comorbidity. To the contrary, incidence in western countries is constant or declining (39). Causes of this are unclear, the coinciding rise of antiosteoporotic treatment is a known and debated factor but does not seem to solely explain the situation (40).

Thus, comorbidity seems to be increasing within a decreasing population. A potential explanation could be increasing preventative measures causing the population to exclusively consist of a high-risk profile population, in terms of a higher morbidity. This coincides with the observed increase in polypharmacy ( $\geq 5$ medications) of $17 \%$ (95\%CI$32 ;-2)$, also being a risk factor (9). Baker et al. found a similar increase of $20 \%$, although defining polypharmacy as $\geq 4$ regular medications. However, studies of the general older population in Sweden have also reported of an increase accordingly and findings in our study could just be reflecting this (41).

Although not significant, results indicate a decrease in PIM-exposure. This resembles decreases seen within the older population in Sweden (42), perhaps bearing witness of increased awareness. Exposure to FRID remained high but psychotropic drugs such as Zolpidem were not as pronounced in 2018 as in 2008, possibly attributable to increased awareness of PIM since these drugs are commonly categorized as both PIM and FRID. Nonetheless, results suggest a lesser awareness regarding FRID, a theory supported by studies reporting of increased prescribing after hip fracture (43).

\section{Malnutrition and sarcopenia}

Since comorbidity has been found a risk factor of frailty and postoperative mortality $(11,13)$ the main expectation would be that an increase would in turn entail concomitant increases of these conditions/outcomes. Our results however propose the opposite. Frailty, a multidimensional clinical condition increasing with age is predictive of falls, disability, hospitalization and death, thus a major issue concerning the population with hip fracture. Fried et al. came up with a definition of frailty in 2001, since then widely used, including weight loss, exhaustion, weakness, slow walking speed and low physical activity (11). Malnutrition, sarcopenia and weight-loss, also widespread syndromes among older people, interrelate with frailty (12) and can therefore grossly serve as indicators. Interestingly, our results presented significantly higher mean values of hand grip strength and CC in 2018 and patients were less likely to have values under cut-off limits for diagnosis of malnutrition and sarcopenia. BMI did not differ, possibly explained by increased knowledge and treatment regarding nutrition, preserving BMI levels in patients otherwise at risk of malnutrition and weight loss due to disease and concomitant loss of appetite. The documentation of weight loss was very poor, especially for cohort 2018, only reported on in 21 of 76 patients, therefore the diagnosis of malnutrition was only based on CC or BMI under cut-off as phenotypic criteria. Results present a significant decrease in malnutrition of $22 \%(95 \% \mathrm{CI}$ $5 ; 37)$, although possibly underestimated in either cohort since weight loss was not included. Additionally, potential effects of oedema and hereditary traits on CC-value cannot be disregarded, possibly causing overestimation. Due to the GLIM-criteria being new there are to our knowledge no direct comparable studies. The prevalence of malnutrition among patients with hip fracture in other studies varies greatly from $<20 \%$ to $>80 \%$, commonly used criteria are low albumin, vitamin D deficiency, BMI $<22 \mathrm{~kg} / \mathrm{m} 2$, weight loss and Mini Nutritional Assessment $(44,45)$.

Sarcopenia also differed significantly with a prevalence of $25 \%$ in cohort 2008 and $11 \%$ in 2018 (95\%CI 1;29), resembling the prevalence of $17 \%$ found in a Spanish study from 2016 using EWGSOP criteria prior to the latest revision (46) and coinciding with the general indication of a decreasing frailty over time. An explanation for a decrease in sarcopenia, malnutrition and possibly frailty could be increased screening, awareness and treatment of the different comorbidities, preventing imminent frailty in older people. Increased awareness as to the importance of physical activity and nutrition is also a possible contributor.

\section{Mortality}

One-year mortality remained unaltered with the rates of $23 \%$ in 2008 compared to $22 \%$ in 2018 (95\% CI $-13 ; 14)$, in line with results of both Jantzen et al reporting of $9.7 \%$ in 1999 compared to $10.3 \%$ in $2012(\mathrm{P}=0.9)$ and Trevisan et al reporting of $25.3 \%$ in 2000 compared to $22.2 \%$ in 2015 $(\mathrm{p}=>0.05)$. In 2008, there was a significant association between ASA-class 3-4 and 1-year mortality (OR 3.5, 95\%CI 1.1;11.6) although the association was not significant for Cohort 2018. This could be attributable to increased individualization of healthcare over time, prioritizing those with greatest need. Coinciding with this, Trevisan et al. found significantly worse Charlson comorbidity index scores (a measure of comorbidity) in survived patients 30 days post-surgery in 2015 compared to in 2000. In addition Brauer et al. and a Danish study comparing patients between 1980-2014 (23) found decreases in shortand long-term mortality irrespective of comorbidity-level, suggesting advances in treatment and rehabilitation of hip fracture.

When adjusted for age, we did not find any statistically significant association between 1-year mortality and malnutrition or sarcopenia in this study. Our relatively small cohort size is a limitation, evident when observing results of previous larger studies. For example, a study including 324 patients with hip fracture found that individuals with sarcopenia had a 1.8 times higher 1-year mortality rate than nonsarcopenic (15). Another larger study on 322 patients with hip fracture, showed that malnutrition was an independent predictor of 1-year mortality (OR 2.4) (14). Despite not being able to show it in this study, malnutrition and sarcopenia most 


\section{MORBIDITY, MALNUTRITION AND SARCOPENIA}

likely have a negative impact on survival and the presented decrease in prevalence could be a major factor contributing to the surprisingly unaltered or even decreasing mortality within a population burdened by increasing morbidity.

\section{Limitations and strengths}

A limitation of this study is the small sample-size, decreasing generalizability. Additionally, data regarding comorbidities and drugs were collected from documentation of ICD-10 codes and drugs in medical records that might have caused an over- or underestimation.

The fact that no exclusion criteria existed is also a limitation since pathological and high-energy-trauma caused hip fractures were included as well, however as seen in table $1,97 \%$ versus $93 \%$ (95\% CI -3;12) had a fracture caused by a coplanar fall.

The dropout group in 2018 had a significantly higher age than the included cohort. Comorbidity is associated with increased age (38), thus results on multimorbidity and medications might have been underestimated in 2018.

The strength of this study is the individually collected data from medical records in combination with individual physical examinations of each patient contributing to a complete evaluation of the population regarding morbidity and frailty in a theoretical as well as a physical sense.

\section{Conclusions}

By comparing two cohorts of patients with hip fracture, a decade apart, our study in line with others suggests an increase in morbidity in terms of increased comorbidityburden and preoperative ASA-class as well as an increase in polypharmacy. One-year mortality rate remained unaltered and results indicated a subsequent decrease in frailty in terms of malnutrition and sarcopenia.

Acknowledgements: The corresponding author, Probert, affirms that she has listed everyone who contributed significantly to the work and has obtained written consent from all contributors who are not authors, named in this section. The study was supported by grants from the Research Committee of Region Örebro, Sweden. Appreciation is expressed to all personnel at the geriatric and orthopaedic wards at Örebro University Hospital who assisted with the collection of data and to Anders Magnuson, Clinical Epidemiology and Biostatistics, Örebro University, for statistical advice.

Author contributions: - Study concept and design: Probert, Akner, Wretenberg, Andersson; - Acquisition of data: Andersson, Probert, Lööw, Johnsson; - Analysis and interpretation of data: Probert, Lööw, Andersson; - Preparation of manuscript: Probert, Lööw, Akner, Wretenberg, Andersson

Ethics declaration: Both projects were approved by the Regional Research and Ethics committee in Uppsala, Sweden, DNR 2008/243 and DNR 2017/490. All included patients were informed about the purpose of the study and signed an informed consent.

Conflict of interest: All authors have no conflicts of interest in this work.

Financial disclosure: All authors have no financial disclosure.

Funding Information: Open access funding provided by Örebro University

Open Access: This article is distributed under the terms of the Creative Commons Attribution 4.0 International License (http://creativecommons.org/licenses/by/4.0/) which permits use, duplication, adaptation, distribution and reproduction in any medium or format, as long as you give appropriate credit to the original author(s) and the source, provide a link to the Creative Commons license and indicate if changes were made.

\section{References}

1. rikshoft_rapport2018_191023.pdf Available at: https://rikshoft.se/wp-content/ uploads/2019/11/rikshoft rapport2018_191023.pdf. Accessed December 11, 2019.

2. Kanis JA, Odén A, McCloskey EV, Johansson H, Wahl DA, Cooper C, IOF Working Group on Epidemiology and Quality of Life. A systematic review of hip fracture incidence and probability of fracture worldwide. Osteoporos Int J Establ Result Coop Eur Found Osteoporos Natl Osteoporos Found USA 2012;23:2239-2256.

3. Be R, Mk K. The Annual Number of Hip Fractures in Sweden Will Double From Year 2002 to 2050: Projections Based on Local and Nationwide Data. Acta Orthop 2014;85. doi:10.3109/17453674.2014.916491.

4. Dyer SM, Crotty M, Fairhall N, Magaziner J, Beaupre LA, Cameron ID, Sherrington C, for the Fragility Fracture Network (FFN) Rehabilitation Research Special Interest Group. A critical review of the long-term disability outcomes following hip fracture. BMC Geriatr 2016;16:158.

5. Hernlund E, Svedbom A, Ivergård M, Compston J, Cooper C, Stenmark J, McCloskey EV, Jönsson B, Kanis JA. Osteoporosis in the European Union: medical management epidemiology and economic burden. Arch Osteoporos 2013;8:136.

6. Roberts SE. Time trends and demography of mortality after fractured neck of femur in an English population, 1968-98: database study. BMJ 2003;327:771-775.

7. Mattisson L, Bojan A, Enocson A. Epidemiology, treatment and mortality of trochanteric and subtrochanteric hip fractures: data from the Swedish fracture register. BMC Musculoskelet Disord 2018;19. doi:10.1186/s12891-018-2276-3.

8. Ritchie CS, Kelley AS, Stijacic Cenzer I, Smith AK, Wallhagen ML, Covinsky KE. High Levels of Geriatric Palliative Care Needs in Hip Fracture Patients Before the Hip Fracture. J Pain Symptom Manage 2016;52:533-538.

9. Lai S-W, Liao K-F, Liao C-C, Muo C-H, Liu C-S, Sung F-C. Polypharmacy Correlates With Increased Risk for Hip Fracture in the Elderly: A Population-Based Study. Medicine (Baltimore) 2010;89:295-299.

10. Reyes C, Estrada P, Nogués X, Orozco P, Cooper C, Díez-Pérez A, Formiga F, Mácias JG, Prieto-Alhambra D. The impact of common co-morbidities (as measured using the Charlson index) on hip fracture risk in elderly men: a population-based cohort study. Osteoporos Int 2014;25:1751-1758.

11. Fried LP, Tangen CM, Walston J, Newman AB, Hirsch C, Gottdiener J, Seeman T, Tracy R, Kop WJ, Burke G, McBurnie MA. Frailty in Older Adults: Evidence for a Phenotype. J Gerontol Ser A 2001;56:M146-M157.

12. A G, D V, E K, M T, S B, Cc S, Y Z. Prevalence and Overlap of Sarcopenia, Frailty, Cachexia and Malnutrition in Older Medical Inpatients. BMC Geriatr 2019;19. doi:10.1186/s12877-019-1115-1.

13. Cher EWL, Allen JC, Howe TS, Koh JSB. Comorbidity as the dominant predictor of mortality after hip fracture surgeries. Osteoporos Int 2019;30:2477-2483.

14. Bell JJ, Pulle RC, Crouch AM, Kuys SS, Ferrier RL, Whitehouse SL. Impact of malnutrition on 12-month mortality following acute hip fracture. ANZ J Surg 201686:157-161

15. Ji Y, H K, Yc H, Hb K, Kh K. Osteosarcopenia in Patients With Hip Fracture Is Related With High Mortality. J Korean Med Sci 2018;33. doi:10.3346/jkms.2018.33. e27.

16. T C, Gl J, Mitd C, et al. GLIM Criteria for the Diagnosis of Malnutrition - A Consensus Report From the Global Clinical Nutrition Community. J Cachexia Sarcopenia Muscle 2019;10. doi:10.1002/jcsm.12383.

17. Cruz-Jentoft AJ, Bahat $\mathrm{G}$, Bauer $\mathrm{J}$, Boirie $\mathrm{Y}$, Bruyère $\mathrm{O}$, Cederholm $\mathrm{T}$, Cooper $\mathrm{C}$, Landi F, Rolland Y, Sayer AA, Schneider SM, Sieber CC, Topinkova E, Vandewoude M, Visser M, Zamboni M, Writing Group for the European Working Group on Sarcopenia in Older People 2 (EWGSOP2) and the EG for E. Sarcopenia: revised European consensus on definition and diagnosis. Age Ageing 2019;48:601-601.

18. Ekstam AK. Mortality, inappropriate medication and length of hospital stay in older hip fracture patients: A general population-based cohort study. 2017;1:7.

19. Ekstam AK, Elmståhl S. Do fall-risk-increasing drugs have an impact on mortality in older hip fracture patients? A population-based cohort study. Clin Interv Aging 2016;11:489.

20. Thorell K, Ranstad K, Midlöv P, Borgquist L, Halling A. Is use of fall risk-increasing drugs in an elderly population associated with an increased risk of hip fracture, after adjustment for multimorbidity level: a cohort study. BMC Geriatr 2014;14. doi:10.1186/1471-2318-14-131.

21. Karampampa K, Ahlbom A, Michaëlsson K, Andersson T, Drefahl S, Modig $\mathrm{K}$. Declining incidence trends for hip fractures have not been accompanied by improvements in lifetime risk or post-fracture survival - A nationwide study of the Swedish population 60years and older. Bone 2015;78:55-61.

22. Brauer CA, Coca-Perraillon M, Cutler DM, Rosen AB. Incidence and Mortality of Hip Fractures in the United States. JAMA 2009;302:1573-1579.

23. Pedersen AB, Ehrenstein V, Szépligeti SK, Lunde A, Lagerros YT, Westerlund A, Tell GS, Sørensen HT. Thirty-five-year Trends in First-time Hospitalization for Hip 


\section{THE JOURNAL OF NUTRITION, HEALTH \& AGING}

Fracture, 1-year Mortality, and the Prognostic Impact of Comorbidity: A Danish Nationwide Cohort Study, 1980-2014. Epidemiol Camb Mass 2017;28:898-905.

24. Jantzen C, Madsen CM, Lauritzen JB, Jørgensen HL. Temporal trends in hip fracture incidence, mortality, and morbidity in Denmark from 1999 to 2012. Acta Orthop 2018;89:170-176.

25. Bekeris J, Wilson LA, Bekere D, Liu J, Poeran J, Zubizarreta N, Fiasconaro M, Memtsoudis SG. Trends in Comorbidities and Complications Among Patients Undergoing Hip Fracture Repair. Anesth Analg, 2019. doi:10.1213/ ANE.0000000000004519.

26. Trevisan C, Gallinari G, Klumpp R, Menon A, Compagnoni R. Year to year comparison of 2000-2015 in hip fracture management: same survival rate despite older and more fragile patients. Aging Clin Exp Res 2019;31:1097-1103.

27. Baker PN, Salar O, Ollivere BJ, Forward DP, Weerasuriya N, Moppett IK, Moran CG Evolution of the hip fracture population: time to consider the future? A retrospective observational analysis. BMJ Open 2014;4:e004405.

28. Mayhew D, Mendonca V, Murthy BVS. A review of ASA physical status - historical perspectives and modern developments. Anaesthesia 2019;74:373-379.

29. Elixhauser A, Steiner C, Harris DR, Coffey RM. Comorbidity measures for use with administrative data. Med Care 1998;36:8-27.

30. Quan H, Sundararajan V, Halfon P, Fong A, Burnand B, Luthi J-C, Saunders LD, Beck CA, Feasby TE, Ghali WA. Coding algorithms for defining comorbidities in ICD-9-CM and ICD-10 administrative data. Med Care 2005;43:1130-1139.

31. Indikatorer för god läkemedelsterapi hos äldre 2017-6-7.pdf Available at: https://www socialstyrelsen.se/globalassets/sharepoint-dokument/artikelkatalog/ovrigt/2017-6-7. pdf. Accessed January 22, 2020.

32. Länkar Bokmärken Hel bok Rek lm 2018-2019 uppdaterad 20190116.pdf Available at: https://www.regionorebrolan.se/Files-sv/\%C3\%96rebro\%20 $1 \% \mathrm{C} 3 \%$ A4ns \%20landsting/V\%C3\%A5rd\%20och\%20h\%C3\%A4lsa/F\%C3\%B6r\%20 v\%C3\%A5rdgivare/L\%C3\%A4kemedelskommitt\%C3\%A9n/Rekommenderade $\% 20$ $1 \% \mathrm{C} 3 \%$ A4kemedel/Rek\%201\%C3\%A4k\%202018-2019/uppdateringar\%20efter\%20 tryckt\%20bok/La\%CC\%88nkar\%20Bokma\%CC\%88rken\%20Hel\%20bok\% 20 Rek\%201m\%202018-2019\%20uppdaterad\%2020190116.pdf. Accessed January 9, 2020 .

33. Aj C-J, G B, J B, Y B, O B, T C, C C, F L, Y R, Aa S, Sm S, Cc S, E T, M V, M V, M Z (2019) Sarcopenia: Revised European Consensus on Definition and Diagnosis. Age Ageing 2019;48. doi:10.1093/ageing/afy169.

34. Vellas B, Guigoz Y, Garry PJ, Nourhashemi F, Bennahum D, Lauque S, Albarede JL. The Mini Nutritional Assessment (MNA) and its use in grading the nutritional state of elderly patients. Nutr Burbank Los Angel Cty Calif 19991;5:116-122.
35. Dodds RM, Syddall HE, Cooper R, et al. Grip Strength across the Life Course: Normative Data from Twelve British Studies. PLOS ONE 2014;9:e113637.

36. Newcombe R, Altman D Proportions and their differences. Statistics with Confidence, London, BMJ Books, pp 45-56.

37. Turesson E, Ivarsson K, Thorngren K-G, Hommel A. The impact of care proces development and comorbidity on time to surgery, mortality rate and functional outcome for hip fracture patients: a retrospective analysis over 19 years with data from the Swedish National Registry for hip fracture patients, RIKSHÖFT. BMC Musculoskelet Disord 2019;20:616.

38. Melis R, Marengoni A, Angleman S, Fratiglioni L. Incidence and Predictors of Multimorbidity in the Elderly: A Population-Based Longitudinal Study. PLOS ONE 2014;9:e103120.

39. Rosengren BE, Björk J, Cooper C, Abrahamsen B. Recent hip fracture trends in Sweden and Denmark with age-period-cohort effects. Osteoporos Int 2017;28:139149

40. Abrahamsen B, Vestergaard P. Declining incidence of hip fractures and the extent of use of anti-osteoporotic therapy in Denmark 1997-2006. Osteoporos Int 2010;21:373380 .

41. Hovstadius B, Hovstadius K, Åstrand B, Petersson G. Increasing polypharmacy - an individual-based study of the Swedish population 2005-2008. BMC Clin Pharmacol 2010;10:16.

42. Hovstadius B, Petersson G, Hellström L, Ericson L. Trends in Inappropriate Drug Therapy Prescription in the Elderly in Sweden from 2006 to 2013: Assessment Using National Indicators. Drugs Aging 2014;31:379.

43. Kragh A, Elmståhl S, Atroshi I. Older Adults' Medication Use 6 Months Before and After Hip Fracture: A Population-Based Cohort Study. J Am Geriatr Soc 2011;59:863-868.

44. Malafarina V, Reginster J-Y, Cabrerizo S, Bruyère O, Kanis J, Martinez J, Zulet M. Nutritional Status and Nutritional Treatment Are Related to Outcomes and Mortality in Older Adults with Hip Fracture. Nutrients 2018;10:555

45. Díaz de Bustamante M, Alarcón T, Menéndez-Colino R, Ramírez-Martín R, Otero Á, González-Montalvo JI. Prevalence of malnutrition in a cohort of 509 patients with acute hip fracture: the importance of a comprehensive assessment. Eur J Clin Nutr 2018;72:77-81.

46. González-Montalvo JI, Alarcón T, Gotor P, Queipo R, Velasco R, Hoyos R, Pardo A, Otero A. Prevalence of sarcopenia in acute hip fracture patients and its influence on short-term clinical outcome. Geriatr Gerontol Int 2016;16:1021-1027. 